\title{
Expression and functional role of Nav1.9 sodium channel in cartwheel cells of the dorsal cochlear nucleus
}

\author{
ZHIYU YAN, YANJUN XU, MIN LIANG and XIAOWEI REN \\ Depatment of Otolaryngology, Beijing Military General Hospital, Beijing 100700, P.R. China
}

Received November 19, 2013; Accepted June 17, 2014

DOI: $10.3892 / \mathrm{mmr} .2014 .2922$

\begin{abstract}
In the central auditory system, cartwheel cells (CWCs) are a group of interneurons in the dorsal cochlear nucleus (DCN). While other DCN neurons respond to stimuli with a simple discharge pattern of single action potentials (SAPs), CWCs respond with complex action potentials (CAPs), consisting of SAPs superimposed on a slow depolarization. The CAPs in CWCs may participate in various auditory or non-auditory signaling processing but its intrinsic mechanisms are largely unknown. In the present study, in vitro whole-cell current clamp recordings on neonatal mice brain slices were used to demonstrate that CWCs respond to brief voltage stimulation with CAPs. Western blotting and immunohistochemistry were also utilized to demonstrate that Nav1.9 was expressed in the CWCs. Finally, when Nav1.9 was genetically silenced, CWCs responded to voltage stimulation with SAPs, not CAPs. The results strongly suggested that Nav1.9 was expressed and functionally contributed to the signaling processing in the central auditory pathway.
\end{abstract}

\section{Introduction}

The interneurons in the dorsal cochlear nucleus (DCN), cartwheel cells (CWCs), are a group of neurons that have crucial roles in processing auditory and non-auditory signals in the brainstem complex (1-3). While other DCN neurons only respond to external stimuli with a simple discharge pattern of single action potentials (SAPs), CWCs respond with complex action potentials (CAPs), consisting of grouped SAPs superimposed on a slow depolarization $(4,5)$.

As CWCs relay inhibitory signals to fusiform cells (FCs), the principle neurons in the DCN forming major projections to higher central auditory pathways, the capacity of CWCs to exhibit a complex discharge pattern has a profound modulatory impact on normal or abnormal sound and sensory transmission

Correspondence to: Dr Zhiyu Yan, Depatment of Otolaryngology, Beijing Military General Hospital, 5 Nanmencang Road, Beijing 100700, P.R. China

E-mail: zhiyuyan01@163.com

Key words: cochlear nucleus, cartwheel cell, Nav1.9 in the brain $(3,6,7)$. However, the exact cellular or molecular mechanisms underlying the intrinsic properties of the complex discharge pattern in CWCs are largely unknown.

Nav1.9 was initially identified by a polymerase chain reaction assay, and was subsequently sequenced and identified as a tetrodotoxin-resistant (TTX-R), slow-inactivating persistent sodium channel $(8,9)$. In spinal cord dorsal root ganglion (DRG) neurons, Nav1.9 channels are highly expressed in nociceptive units, and contribute to non-inactivating and persistent depolarization of membrane potentials of the neurons, therefore regulating signaling transduction between central and peripheral nociceptive pathways (9-11). In the present study, western blotting and immunohistochemistry were utilized to examine the expression of Nav1.9 in dorsal cochlear CWCs. Furthermore, electrophysiology along with gene silencing were applied to examine the cellular mechanisms of Nav1.9 in contributing to the intrinsic action potential firing patterns of CWCs in the central auditory pathway.

\section{Materials and methods}

Brain slices. The present study was approved by the Ethics Committee of the Department of Otolaryngology, Beijing Military General Hospital (Beijing, China). C57BL/6 mice (postnatal 12-14 days; The Jackson Laboratory, Bar Harbor, ME, USA) were anesthetized with subcutaneous injections of a mixture of ketamine $(50 \mathrm{mg} / \mathrm{kg})$ and xylazine $(50 \mathrm{mg} / \mathrm{kg})$ administered in the abdominal area. The mice were decapitated, and the cortex and cerebellum were removed to expose the brainstem. The brainstem was then quickly separated and placed in a glass petri dish filled with ice-cold artificial cerebrospinal fluid (ACSF), containing $130 \mathrm{mM} \mathrm{NaCl}, 3 \mathrm{mM} \mathrm{KCl}$, $1.25 \mathrm{mM} \mathrm{KH}_{2} \mathrm{PO}_{4}, 20 \mathrm{mM} \mathrm{NaHCO}, 10 \mathrm{mM}$ glucose, $2.5 \mathrm{mM} \mathrm{CaCl}_{2}$ and $1.3 \mathrm{mM} \mathrm{MgSO}_{4}$. A vibratory microtome (Vibratome; Oxford Instruments Microspec, San Mateo, CA, USA) was used to cut 200- $\mu \mathrm{m}$ trans-strial slices, which were maintained in ACSF supplemented with $5 \% \mathrm{CO}_{2}$ at $30^{\circ} \mathrm{C}$.

Electrophysiology. The in vitro whole-cell current clamp electrophysiology was conducted on a recording chamber mounted on an Olympus BX51W1 upright microscope (Olympus Corporation, Tokyo, Japan) with a $63 \mathrm{X}, 0.8$ N.A. water immersion objective. The recording was conducted by a Multiclamp 700A amplifier (Axon Instruments, Foster City, CA, USA), filtered at $5 \mathrm{KHz}$ and digitized at $50 \mathrm{KHz}$ with a 12-bit A/D digitizer (National 
Instruments Corporation, Austin, TX, USA). The brain slices were maintained in the recording chamber with continuous incubation with ACSF $\left(2-4 \mathrm{ml} / \mathrm{min}, 95 \% \mathrm{O}_{2}\right.$ and $5 \% \mathrm{CO}_{2}$ to maintain a $\mathrm{pH}$ level of 7.1-7.5). The recording pipette solution contained $120 \mathrm{~mm}$ potassium gluconate, $20 \mathrm{~mm} \mathrm{KCl}, 2 \mathrm{~mm}$ sodium phosphocreatine, $4 \mathrm{~mm}$ MgATP, $10 \mathrm{~mm}$ HEPES, $0.3 \mathrm{~mm}$ NaGTP and $1.1 \mathrm{~mm}$ EGTA, maintained at $\mathrm{pH} 7.2$ with $10 \mathrm{~mm} \mathrm{KOH}$. For the pharmacological ion-channel antagonists, $100 \mu \mathrm{M}$ $\mathrm{CdCl}_{2}$ was used to block the voltage-gated $\mathrm{Ca}^{2+}$ channels and $100 \mathrm{~nm}$ TTX to block the fast-inactivating sodium channels.

Western blotting. For western blotting analysis, the brain slices of cochlea nucleus were further micro-dissected under the microscope to separate the superficial layer, mainly consisting of CWCs, from the molecular layer, mainly constituting pyramidal neurons. After being quickly frozen on dry ice, RIPA buffer, containing $150 \mathrm{~mm} \mathrm{NaCl}, 50 \mathrm{~mm}$ Tris, $1 \%$ Triton X-100, $0.1 \%$ sodium dodecyl sulfate and $1 \%$ Nadeoxycholate $(\mathrm{pH} 7.4)$ was used to collect brain tissue. A Bio-Rad protein assay (Bio-Rad Laboratories, Hercules, CA, USA) was then applied to equal the protein concentrations between the two types of samples. The protein lysates were resolved by sodium dodecyl sulfate-polyacrylamide gel electrophoresis, followed by transfer onto nitrocellulose membranes (Amersham Biosciences). The blocking medium was phosphate-buffered saline (PBS) containing $0.2 \%$ Tween- 20 and $5 \%$ non-fat dry milk. The lysates were then incubated with rabbit anti-Nav1.9/NaN primary antibody (1:1,000; Alomone Laboratories Ltd., Jerusalem, Israel) and goat anti-rabbit horseradish peroxidase-labeled secondary antibody, as detected by X-ray film.

Immunohistochemistry. The superficial layer of the cochlear nucleus slices were dissociated and plated in 6-well plates. After $1 \mathrm{~h}$, the dissociated cells were fixed with $4 \%$ paraformaldehyde in $10 \mathrm{~mm}$ phosphate buffer $(\mathrm{PB})$ at room temperature for $1 \mathrm{~h}$. Next, the cells were incubated for $1 \mathrm{~h}$ in $0.05 \%$ Triton X-100 and $10 \%$ goat serum in PBS at room temperature, followed by incubation with primary antibody (rabbit anti-Nav1.9/NaN; $1: 100$ ) at $4^{\circ} \mathrm{C}$ overnight. Following three washes with PBS, the cells were incubated with polyclonal goat-anti-rabbit IgG secondary antibody (Alexa 488; 1:500; Invitrogen Life Technologies, Carlsbad, CA, USA) for $2 \mathrm{~h}$ at room temperature. The bottoms of the plates were then mounted with glass cover slips to prevent detachment, then imaged with a confocal microscopy (Olympus Corporation).

Nav1.9 gene silencing. Mouse Nav1.9 (SCN11A) small interfering (si)RNA and scrambled siRNA (negative control) were purchased from Integrated DNA Technologies, Inc. (Coralville, IL, USA). Cochlear nucleus slices were transfected with SCN11A-siRNA $(100 \mathrm{~nm})$ or the scrambled siRNA using GeneSilencer (Genlantis, San Diego, CA, USA) according to the manufacturer's instructions. Following transfection, the slices were incubated in a petri dish containing Dulbecco's modified Eagle's medium, 10\% fetal bovine serum, N2 and B27 supplements (Invitrogen Life Technologies) at $37^{\circ} \mathrm{C}$ in 5\% $\mathrm{CO}_{2}$, overnight prior to electrophysiology.

Statistical analysis. Student's t test was performed to determine significance, using SPSS version 11.0 software (SPSS
A



B

Control $\quad \mathrm{Cd}^{2+} \quad \mathrm{Cd}^{2+}+\mathrm{TTX}$

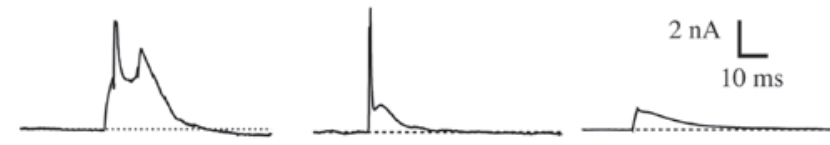

Figure 1. Electrophysiological properties of CWCs in the dorsal cochlear nucleus. (A) In an in vitro whole-cell current-clamp recording, a CWC responded to injected current stimulus with a firing pattern characteristic of CAPs. (B) A CWC responded to a suprathreshold current injection with CAPS $(l e f t)$. Action potentials were not completely blocked by the $\mathrm{Ca}^{2+}$ channel antagonist $\mathrm{Cd}^{2+}(100 \mu \mathrm{M}$; middle), and a slow depolarization persisted following the application of $\mathrm{Cd}^{2+}$ and TTX $(100 \mathrm{~nm})$, a fast-inactivating sodium channel antagonist (right). CWC, cartwheel cells; CAPS, complex action potentials; TTX, tetrodotoxin.

Inc., Chicago, IL, USA). $\mathrm{P}<0.05$ was considered to indicate a statistically significant difference.

\section{Results}

Slow depolarization of CAPs in CWCs is resistant to $\mathrm{Ca}^{2+} / \mathrm{Na}^{+}$ antagonists. In vitro whole-cell current-clamp recordings were conducted on 122 neurons on the superficial layer of the cochlear nucleus slides. The cells were initially held with holding potentials of $\sim-70 \mathrm{mV}$ and $150 \mathrm{msec}$ of current injections were used to generate suprathreshold responses from the cells. Among them, 84 of the recorded neurons demonstrated characteristic suprathreshold responses of CWCs, which were CAPs, consisting of trains of SAPs superimposed on slow depolarizations (Fig. 1A).

In order to identify the intrinsic sub-types of the ion channels contributing to CAPs in CWCs, short-stimuli current clamp recordings (5 msec current injections) were then utilized, along with pharmacological reagents, to block specific ion channels. The results demonstrated that under control conditions, the suprathreshold current injection elicited CAPs consisting of two SAPs on a slow-rising depolarization (Fig. 1B, left). Notably, while the voltage-gated calcium channel antagonist $\mathrm{Cd}^{2+}(100 \mu \mathrm{M})$ was applied in the bath medium of ACSF, the blockage of $\mathrm{Ca}^{2+}$ currents did not eliminate the slow depolarization. Instead it resulted in a decreased slow depolarization with a SAP superimposed on it (Fig. 1B, middle). Further application of TTX (100 nm), the antagonist of fast-inactivating sodium channels, blocked SAPs but did not block the slow depolarization.

Therefore, the results demonstrated that ionic currents, other than calcium currents and TTX-sensitive fast-inactivating sodium currents, contributed to the slow depolarizations of CAPs in CWCs. 
A

$\begin{array}{ll}\begin{array}{ll}\text { Fusiform } \\ \text { layer }\end{array} & \begin{array}{l}\text { Superficial } \\ \text { layer }\end{array}\end{array}$

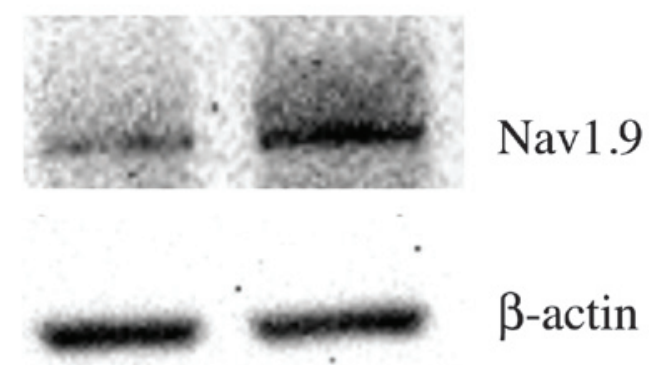

B

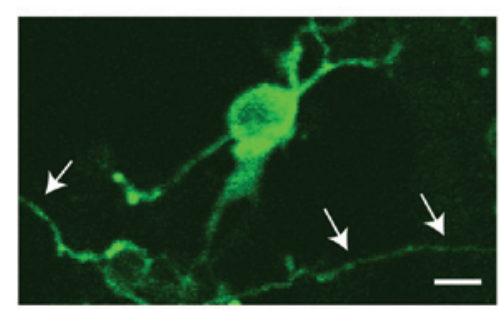

Figure 2. Nav1.9 was expressed in CWCs. (A) Western blot analysis was conducted on fusiform layer tissues and superficial layer tissues of the dorsal cochlear nucleus with a Nav1.9 antibody. $\beta$-actin served as the control. (B) Immunohistochemistry revealed a neuron in the superficial layer exhibited positive expression of Nav1.9. It was determined to be a CWC due to its morphologic characterization of curving dendrites (arrows). Scale bar, $10 \mu \mathrm{M}$. CWC, cartwheel cells.

A

SCN11A-SiRNA

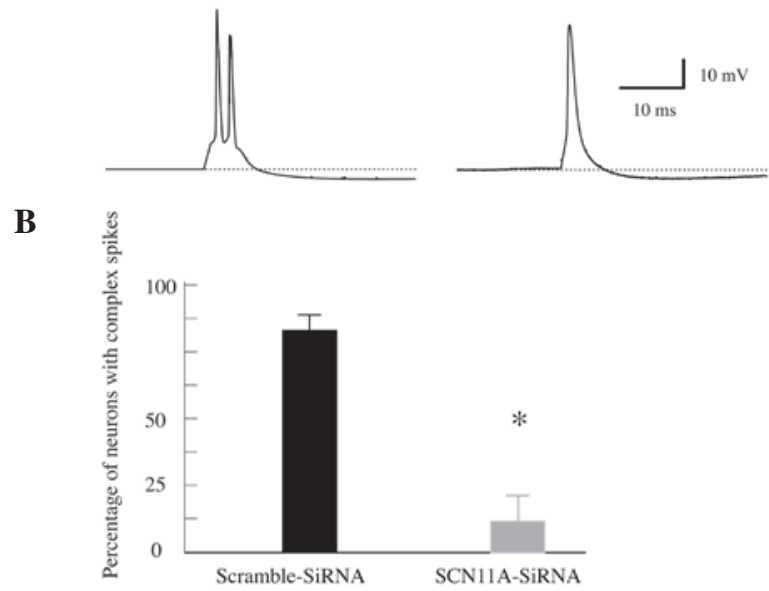

Figure 3. Genetically silencing Nav1.9 converted CAPs to SAPs. (A) In whole-cell current-clamp recordings with the blockage of $\mathrm{Ca}^{2+}$ currents, a CWC responded to suprathreshold stimuli with CAPs with the application of non-specific scramble-siRNA (100 nm; left), whereas another CWC responded with a SAP following SCN11A-siRNA transfection $(100 \mathrm{~nm}$; right). (B) The percentage of CWCs firing CAPs was significantly reduced with the genetic silencing of the Nav1.9 gene. ${ }^{*} \mathrm{P}<0.05$, compared with scramble-siRNA. CAPs, complex action potentials; SAPs, single action potentials; CWCs, cartwheel cells; siRNA, small interfering RNA.

Expression of Nav1.9 in CWCs. Other experimental methods were then utilized to determine the identity of the ion channels contributing to the signature firing pattern of CAP in CWCs.
Following micro-array screening of ion channel expression in the cochlear nucleus, it was noted that Nav1.9, a TTX-resistant slow-inactivating sodium channel was likely to be expressed in the dorsal cochlear nucleus. Then, western blot analysis was used to confirm whether Nav1.9 was expressed in the dorsal cochlear nucleus or even CWCs. Following micro-dissection, the superficial layer, which mainly consisted of CWCs from the dorsal cochlear nucleus, was further separated from the fusiform layer (which mainly consisted of principal pyramidal neurons) on the cochlear nucleus brainstem slides. The two types of tissues then underwent western blot analysis using an antibody against Nav1.9. The results demonstrated that, as compared with the fusiform layer, the superficial layer had significantly stronger reaction to the Nav1.9 antibody (Fig. 2A), suggesting that the TTX-resistant slow-inactivating Nav1.9 $\mathrm{Na}^{+}$channel was highly likely to be expressed in CWCs, the major cellular population in the superficial layer of the dorsal cochlear nucleus.

The western blot analysis result was also confirmed by immunohistochemistry. The superficial layer was dissociated with trituration and the dissociated cells were plated on 6-well plates. After the application of a Nav1.9 primary antibody followed by a fluorescent secondary antibody, positive staining was observed in a number of the cells (Fig. 2B). Based on morphological characterizations, those Nav1.9-positive cells had cell body sizes of $\sim 20 \mu \mathrm{M}$ and their dendrites branched at wide angles or even curved back toward the somas, and were therefore positively identified as CWCs from the dorsal cochlear nucleus (Fig. 2B) (12).

Nav1.9 functionally contributes to the firing of CAPs in CWCs. Finally, the functional role of Nav1.9 in contributing to the ionic properties in CWCs was examined by a loss-of-function siRNA genetic silencing assay. Brainstem slices containing dorsal cochlear nucleus were treated with either Nav1.9 siRNA (SCN11A-siRNA, $100 \mathrm{~nm}$ ), or a non-specific control siRNA (scramble-siRNA, $100 \mathrm{~nm}$ ). Twenty-four hours later, whole-cell current-clamp recordings were conducted on the neurons in the superficial layer of the dorsal cochlear nucleus with the addition of $\mathrm{Cd}^{2+}$ in the bath medium to block voltage-gated $\mathrm{Ca}^{2+}$ channels.

Subesquent to genetically silencing Nav1.9 in the cochlear nucleus, it was identified that the majority of the neurons from superficial layer, presumably CWCs, responded to suprathreshold current stimuli with SAPs, not CAPs (Fig. 3A). The statistical analysis demonstrated that $87 \pm 4 \%$ of the recorded CWCs fired complex spikes in the brainstem slices treated with non-specific scramble-siRNA, whereas only $11 \pm 6 \%$ of the neurons fired complex spikes in the slices treated with Nav1.9 siRNA ( $\mathrm{P}<0.05$; Fig. 3B). Therefore, the present results strongly suggest that Nav1.9 functionally contributed to the generation of CAPs in CWCs in the dorsal cochlear nucleus.

\section{Discussion}

In the brainstem cochlear nucleus, CWCs are the only group of neurons that fire CAPs in response to suprathreshold stimuli. This unique electrophysiological property of CWCs undoubtedly has profound effects on the signaling processing in auditory and non-auditory pathways in 
the brain. Until now, little has been determined regarding the intrinsic ionic mechanisms contributing to the generation of CAPs in CWCs. In the present study, it was demonstrated that a persistent, slow-inactivating and TTX-resistant $\mathrm{Na}^{+}$channel subtype, Nav1.9, was present in the CWCs and was functionally responsible for the firing pattern of CAPs.

In other neural populations, persistent $\mathrm{Na}^{+}$currents are associated with various patterns of action potentials, including spontaneous action potentials (13-16), activation of afterdepolarization (17-19) or even subthreshold membrane potential oscillations (20-22). In dorsal cochlear CWCs, the neurons do not fire subthreshold oscillations or spontaneous action potentials, although a low frequency of spontaneous activity has previously been recorded from the cochlear nucleus of decerebrate cats under pathological conditions (23). Therefore, it appears that a persistent $\mathrm{Na}^{+}$current in CWCs functionally contributes to the generation of after-depolarizations or subthreshold slow depolarizations, as demonstrated by the evidence that genetic silencing of Nav1.9 eliminated after-depolarizations, therefore converted CAPs into SAPs (Fig. 3). Notably, other ionic currents, including TTX-sensitive fast-inactivating $\mathrm{Na}^{+}$current and voltage-gated $\mathrm{Ca}^{2+}$ current also contributed to the generation of CAPs in CWCs, as demonstrated in our recordings with pharmacological antagonists to those ion channels. Therefore, it is highly likely that the delicate ionic homeostasis or the balance of ion concentrations of various cations, including $\mathrm{Na}^{+}$ and $\mathrm{Ca}^{2+}$ or $\mathrm{K}^{+}$, may be decisive in generating complex or simple firing patterns in CWCs.

In conclusion, to the best of our knowledge, the present study is the first report the presence and functional role of persistent $\mathrm{Na}^{+}$channels in the dorsal cochlear nucleus. The results undoubtedly further the understanding of the underlying cellular and molecular mechanisms of the neural circuits in the auditory central nervous system.

\section{References}

1. Kuo SP and Trussell LO: Spontaneous spiking and synaptic depression underlie noradrenergic control of feed-forward inhibition. Neuron 71: 306-318, 2011.

2. Oertel D and Young ED: What's a cerebellar circuit doing in the auditory system? Trends Neurosci 27: 104-110, 2004.

3. Kaltenbach JA: The dorsal cochlear nucleus as a participant in the auditory, attentional and emotional components of tinnitus. Hear Res 216-217: 224-234, 2006.

4. Manis PB, Spirou GA, Wright DD, Paydar S and Ryugo DK: Physiology and morphology of complex spiking neurons in the guinea pig dorsal cochlear nucleus. J Comp Neurol 348: 261-276, 1994.

5. Zhang S and Oertel D: Cartwheel and superficial stellate cells of the dorsal cochlear nucleus of mice: intracellular recordings in slices. J Neurophysiol 69: 1384-1397, 1993.
6. Caspary DM, Hughes LF, Schatteman TA and Turner JG: Age-related changes in the response properties of cartwheel cells in rat dorsal cochlear nucleus. Hear Res 216-217: 207-215, 2006.

7. Smith PF: The Endocannabinoid System in the Cochlear Nucleus and Its Implications for Tinnitus Treatment. In: Textbook of Tinnitus. Springer New York, NY, pp639-647, 2011.

8. Dib-Hajj SD, Tyrrell L, Black JA and Waxman SG: NaN, a novel voltage-gated $\mathrm{Na}$ channel, is expressed preferentially in peripheral sensory neurons and down-regulated after axotomy. Proc Natl Acad Sci USA 95: 8963-8968, 1998.

9. Tate S, Benn S, Hick C, et al: Two sodium channels contribute to the TTX-R sodium current in primary sensory neurons. Nat Neurosci 1: 653-655, 1998.

10. Herzog RI, Cummins TR and Waxman SG: Persistent TTX-resistant $\mathrm{Na}^{+}$current affects resting potential and response to depolarization in simulated spinal sensory neurons. J Neurophysiol 86: 1351-1364, 2001.

11. Amaya F, Decosterd I, Samad TA, et al: Diversity of expression of the sensory neuron-specific TTX-resistant voltage-gated sodium ion channels SNS and SNS2. Mol Cell Neurosci 15: $331-342,2000$.

12. Wouterlood FG and Mugnaini E: Cartwheel neurons of the dorsal cochlear nucleus: a Golgi-electron microscopic study in rat. J Comp Neurol 227: 136-157, 1984.

13. Kononenko NI, Shao LR and Dudek FE: Riluzole-sensitive slowly inactivating sodium current in rat suprachiasmatic nucleus neurons. J Neurophysiol 91: 710-718, 2004.

14. Shuai J, Bikson M, Hahn PJ, Lian J and Durand DM: Ionic mechanisms underlying spontaneous CA1 neuronal firing in $\mathrm{Ca}^{2+}$-free solution. Biophys J 84: 2099-2111, 2003.

15. Taddese A and Bean BP: Subthreshold sodium current from rapidly inactivating sodium channels drives spontaneous firing of tuberomammillary neurons. Neuron 33: 587-600, 2002.

16. Yamada-Hanff J and Bean BP: Persistent sodium current drives conditional pacemaking in CA1 pyramidal neurons under muscarinic stimulation. J Neurosci 33: 15011-15021, 2013.

17. Bouhadfane M, Tazerart S, Moqrich A, Vinay L and Brocard F: Sodium-mediated plateau potentials in lumbar motoneurons of neonatal rats. J Neurosci 33: 15626-15641, 2013.

18. Yue C, Remy S, Su H, Beck H and Yaari Y: Proximal persistent $\mathrm{Na}^{+}$channels drive spike afterdepolarizations and associated bursting in adult CA1 pyramidal cells. J Neurosci 25: 9704-9720, 2005.

19. D'Ascenzo M, Podda MV, Fellin T, et al: Activation of mGluR5 induces spike afterdepolarization and enhanced excitability in medium spiny neurons of the nucleus accumbens by modulating persistent $\mathrm{Na}^{+}$currents. J Physiol 587: 3233-3250, 2009.

20. Xie RG, Zheng DW, Xing JL, et al: Blockade of persistent sodium currents contributes to the riluzole-induced inhibition of spontaneous activity and oscillations in injured DRG neurons. PloS One 6: e18681, 2011.

21. Ziskind-Conhaim L, Wu L and Wiesner EP: Persistent sodium current contributes to induced voltage oscillations in locomotor-related hb9 interneurons in the mouse spinal cord. J Neurophysiol 100: 2254-2264, 2008.

22. Dong H, Fan YH, Wang YY, Wang WT and Hu SJ: Lidocaine suppresses subthreshold oscillations by inhibiting persistent $\mathrm{Na}^{(+)}$ current in injured dorsal root ganglion neurons. Physiol Res 57: 639-645, 2008.

23. Parham K and Kim DO: Spontaneous and sound-evoked discharge characteristics of complex-spiking neurons in the dorsal cochlear nucleus of the unanesthetized decerebrate cat. J Neurophysiol 73: 550-561, 1995. 